\title{
The composition of extended Mittag-Leffler functions with pathway integral operator
}

\author{
G Rahman ${ }^{1 *}$, A Ghaffar², S Mubeen ${ }^{3}$, M Arshad $^{1}$ and SU Khan ${ }^{4}$
}

\section{"Correspondence:}

gauhar55uom@gmail.com

'Department of Mathematics,

International Islamic University, Islamabad, Pakistan

Full list of author information is

available at the end of the article

\begin{abstract}
In this paper, we present certain composition formulae of the pathway fractional integral operators associated with two extended Mittag-Leffler functions. Here, we find out the relevant connections of some particular cases of the main results with those earlier ones.
\end{abstract}

Keywords: extended Mittag-Leffler function; pathway fractional integral operator

\section{Introduction}

Mittag-Leffler functions play a vital role in determining the solutions of fractional differential and integral equations which are associated with an extensive variety of problems in diverse areas of mathematics and mathematical physics. In addition, from exponential behavior, the deviations of physical phenomena could also be represented by means of Mittag-Leffler functions. Therefore, the uses of Mittag-Leffler functions are constantly rising, especially in physics. For more details about the recent research in the field of dynamical systems theory, stochastic systems, non-equilibrium statistical mechanics and quantum mechanics, the readers may refer to the recent work of the researchers [1-5] and the references cited therein.

Now, we begin with the Mittag-Leffler functions $E_{\alpha}(z)$ and $E_{\alpha, \beta}(z)$ defined in the form of the following series:

$$
E_{\alpha}(z)=\sum_{n=0}^{\infty} \frac{z^{n}}{\Gamma(\alpha n+1)}, \quad z \in \mathbb{C} ; \Re(\alpha)>0
$$

and

$$
E_{\alpha, \beta}(z)=\sum_{n=0}^{\infty} \frac{z^{n}}{\Gamma(\alpha n+\beta)}, \quad z, \beta \in \mathbb{C} ; \Re(\alpha)>0
$$

respectively. For further study of Mittag-Leffler functions like generalizations and applications, the readers may refer to the work of researchers [6-10], Kilbas et al. [11], and Saigo and Kilbas [12]. In recent years, the Mittag-Leffler function (1) and some of its generalizations have been numerically established in the complex plane [13, 14]. A new general-

(c) The Author(s) 2017. This article is distributed under the terms of the Creative Commons Attribution 4.0 International License (http://creativecommons.org/licenses/by/4.0/), which permits unrestricted use, distribution, and reproduction in any medium, provided you give appropriate credit to the original author(s) and the source, provide a link to the Creative Commons license, and indicate if changes were made. 
ization of the Mittag-Leffler functions $E_{\alpha, \beta}(z)$ in (2) has been defined by Prabhakar [15] as given below:

$$
E_{\alpha, \beta}^{\gamma}(z)=\sum_{n=0}^{\infty} \frac{(\gamma)_{n}}{\Gamma(\alpha n+\beta)} \frac{z^{n}}{n !}, \quad z, \beta \in \mathbb{C} ; \mathfrak{i}(\alpha)>0,
$$

where $(\gamma)_{n}$ denotes the well-known Pochhammer's symbol which is defined by

$$
(\gamma)_{n}= \begin{cases}1 & (n=0, \gamma \in \mathbb{C}), \\ \gamma(\gamma+1) \cdots(\gamma+n-1) & (n \in \mathbb{N}, \gamma \in \mathbb{C}) .\end{cases}
$$

In fact, the following special cases are satisfied:

$$
E_{\alpha, \beta}^{1}(z)=E_{\alpha, \beta}(z)=E_{\alpha, 1}^{1}(z)=E_{\alpha}(z)
$$

Many researchers have established the significance and great consideration of MittagLeffler functions in the theory of special functions to explore the generalizations and some applications. Various extensions for these functions are found in [16-20]. Srivastava and Tomovski [21] defined the further generalization of the Mittag-Leffler function $E_{\alpha, \beta}^{\gamma}(z)$ as given in (3), which is defined as

$$
E_{\alpha, \beta}^{\gamma, \kappa}(z)=\sum_{n=0}^{\infty} \frac{(\gamma)_{n \kappa}}{\Gamma(\alpha n+\beta)} \frac{z^{n}}{n !}
$$

where $z, \beta, \gamma \in \mathbb{C} ; \mathfrak{R}(\alpha)>\max \{0, \mathfrak{R}(\kappa)-1\} ; \mathfrak{R}(\kappa)>0$.

Özarslan and Yilmaz [22] investigated an extended Mittag-Leffler function $E_{\alpha, \beta}^{\gamma ; c}(z ; p)$, which is defined as

$$
E_{\alpha, \beta}^{\gamma ; c}(z ; p)=\sum_{n=0}^{\infty} \frac{B_{p}(\gamma+n, c-\gamma)}{B(\gamma, c-\gamma)} \frac{(c)_{n}}{\Gamma(\alpha n+\beta)} \frac{z^{n}}{n !},
$$

where $p \geq 0, \mathfrak{R}(c)>\Re(\gamma)>0$ and $B_{p}(x, y)$ is an extended beta function defined in [23, 24] as follows:

$$
B_{p}(x, y)=\int_{0}^{1} t^{x-1}(1-t)^{y-1} e^{-\frac{p}{t(1-t)}} d t
$$

where $\Re(p)>0, \Re(x)>0$ and $\Re(y)>0$. If $p=0$, then the function $B_{p}(x, y)$ reduces to the following beta function:

$$
B(x, y)=\int_{0}^{1} t^{x-1}(1-t)^{y-1} d t .
$$

The gamma function is defined by

$$
\Gamma(z)=\int_{0}^{\infty} t^{z-1} e^{-t} d t ; \quad \Re(z)>0 .
$$


By inspection, we conclude the following relation:

$$
\Gamma(z+1)=z \Gamma(z)
$$

Mittal et al. [25] defined an extended generalized Mittag-Leffler function as

$$
E_{\alpha, \beta}^{\gamma, q ; c}(z ; p)=\sum_{n=0}^{\infty} \frac{B_{p}(\gamma+n q, c-\gamma)}{B(\gamma, c-\gamma)} \frac{(c)_{n q}}{\Gamma(\alpha n+\beta)} \frac{z^{n}}{n !}
$$

where $\alpha, \beta, \gamma \in \mathbb{C}, \Re(\alpha)>0, \Re(\beta)>0, \Re(\gamma)>0, q>0$ and $B_{p}(x, y)$ is an extended beta function defined in (7).

The fractional calculus is a field of applied mathematics that deals with the fractional derivatives and fractional integrals of arbitrary orders. During the last few decades, many researchers have applied fractional calculus to all fields of science such as engineering and mathematics. The researchers have developed a significant contributions in the field of fractional calculus such as fractional derivatives of constant and variable orders, global existence solution of differential equations; an alternative method for solving generalized differential equations of fractional order, a new type of fractional derivative formula containing the normalized sine function without singular kernel. For the recent development in the field of fractional calculus, the readers are referred to the work of [26-31] and [3235].

Recently, Nair [36] introduced a pathway fractional integral operator by using the idea of Mathai [37], and Mathai and Haubold [38, 39], which is defined as

$$
\left(P_{0+}^{\mu, \lambda} f\right)(x)=x^{\mu} \int_{0}^{\left[\frac{x}{\alpha(1-\lambda)}\right]}\left[1-\frac{\alpha(1-\lambda) \tau}{x}\right]^{\frac{\mu}{(1-\lambda)}} f(\tau) d \tau
$$

where $f \in L(a, b)(L(a, b)$ is a Lebesgue measurable real or complex-valued function), $\mu \in \mathbb{C}, \Re(\mu)>0, \alpha>0$ and $\lambda<1$ ( $\lambda$ is a pathway parameter) (cf. [40]). For a given scalar $\lambda$ and scalar random variables, the pathway model is defined by the following probability density function:

$$
f(x)=c|x|^{\nu-1}\left[1-\alpha(1-\lambda)|x|^{\eta}\right]^{\frac{\mu}{(1-\lambda)}},
$$

where $x \in(-\infty, \infty) ; \eta>0 ; \mu>0 ;\left[1-\alpha(1-\lambda)|x|^{\eta}\right]^{\frac{\mu}{(1-\lambda)}}>0 ; v>0$ and where $c$ and $\lambda$ denote the normalizing constant and pathway parameter, respectively.

Moreover, the normalizing constants for $\lambda \in \mathbb{R}$ are defined by the following:

$$
c= \begin{cases}\frac{1}{2} \frac{\eta[\alpha(1-\lambda)]^{\frac{v}{\eta}} \Gamma\left(\frac{v}{\eta}+\frac{\mu}{\lambda-1}+1\right)}{\Gamma\left(\frac{v}{\eta}\right) \Gamma\left(\frac{1}{1-\lambda}+1\right)} & (\lambda<1), \\ \frac{1}{2} \frac{\eta[\alpha(1-\lambda)]^{\frac{v}{\eta}} \Gamma\left(\frac{\mu}{\lambda-1}\right)}{\Gamma\left(\frac{v}{\eta}\right) \Gamma\left(\frac{\mu}{\lambda-1}-\frac{v}{\eta}\right)} & \left(\frac{1}{\lambda-1}-\frac{v}{\eta}>0, \lambda>1\right), \\ \frac{1}{2} \frac{(\alpha \mu) \frac{\nu}{\eta}}{\Gamma\left(\frac{\nu}{\eta}\right)} & (\lambda \rightarrow 1) .\end{cases}
$$

It is noted that if $\lambda<1$, we have $\left[1-\alpha(1-\lambda)|x|^{\eta}\right]^{\frac{\mu}{(1-\lambda)}}>0$, and (13) can be considered a member of the extended generalized type-1 beta family. Also the extended type- 
1 beta density, the triangular density, the uniform density and many other probability density functions are specific cases of the pathway density function defined in (13) for $\lambda<1$.

For instance, if $\lambda>1$, and by putting $(1-\lambda)=-(\lambda-1)$ in (12), then it yields

$$
\left(P_{0+}^{\mu, \lambda} f\right)(x)=x^{\mu} \int_{0}^{\left[\frac{x}{-\alpha(1-\lambda)}\right]}\left[1+\frac{\alpha(\lambda-1) \tau}{x}\right]^{\frac{\mu}{-(\lambda-1)}} f(\tau) d \tau
$$

and

$$
f(x)=c|x|^{\nu-1}\left[1+\alpha(\lambda-1)|x|^{\eta}\right]^{-\frac{\mu}{(\lambda-1)}}
$$

where $x \in(-\infty, \infty) ; \eta>0 ; \mu>0$; and $\lambda>1$ represents the extended generalized type- 2 beta model for real $x$. The type- 2 beta density function, the $F$ density function, the student $t$ density function and many other density functions are particular cases of the density function defined in (15). Furthermore, if $\lambda \rightarrow 1_{-}$, then (12) reduces to the Laplace integral transform. Similarly, if $\lambda=0, \alpha=1$ and $\mu$ is replaced by $\mu-1$, then (12) reduces to the well-known Riemann-Liouville fractional integral operator.

The pathway fractional integral operator (12) leads to several other interesting examples such as fractional calculus related to several probability density functions and their applications in statistical theory. Nisar et al. [41] presented the pathway fractional integral formulae associated with Struve function of the first kind. The results given in [40] are a slight generalization of the result provided by Agarwal and Purohit [42] and Nair [36]. Recently, Nisar et al. [43] provided the composition of pathway integral operator associated with generalized $k$-Mittag-Leffler functions. The main aim of this study is to obtain pathway fractional integral operators associated with extended Mittag-Leffler functions.

\section{Pathway fractional integration of an extended Mittag-Leffler function}

In this section, we derive the pathway integration formulae involving the extended MittagLeffler functions from (6).

Theorem 1 Suppose that $\rho ; \beta ; \gamma ; c ; \mu \in \mathbb{C} ;\{\Re(\rho) ; \Re(\beta) ; \Re(\mu) ; \Re(c)\}>0 ; \Re\left(\frac{\mu}{1-\lambda}\right)>-1 ; \lambda<1$; $p \geq 0$ and $\omega \in \mathbb{R}$. Then the following formula holds true:

$$
P_{0+}^{\mu, \lambda}\left[\tau^{\beta-1} E_{\rho, \beta}^{\gamma ; c}\left[\left(\omega \tau^{\rho}\right) ; p\right]\right](x)=\frac{\Gamma\left(1+\frac{\mu}{1-\lambda}\right) x^{\mu+\beta}}{[\alpha(1-\lambda)]^{\beta}} E_{\rho, \beta+\left(1+\frac{\mu}{1-\lambda}\right)}^{\gamma ; c}\left[\omega\left(\frac{x}{\alpha(1-\lambda)}\right)^{\rho} ; p\right] .
$$

Proof By using (6) and (12), we have

$$
P_{0+}^{\mu, \lambda}\left[\tau^{\beta-1} E_{\rho, \beta}^{\gamma ; c}\left[\left(\omega \tau^{\rho}\right) ; p\right]\right](x)=x^{\mu} \int_{0}^{\left[\frac{x}{\alpha(1-\lambda)}\right]} \tau^{\beta-1}\left[1-\frac{\alpha(1-\lambda) \tau}{x}\right]^{\frac{\mu}{1-\lambda}} E_{\rho, \beta}^{\gamma ; c}\left(\omega \tau^{\rho} ; p\right) d \tau .
$$


We denote the right-hand side of the above equation by $I_{1}$, and after interchanging the order of integration and summation, we have

$$
\begin{aligned}
I_{1} & =x^{\mu} \int_{0}^{\left[\frac{x}{\alpha(1-\lambda)}\right]} \tau^{\beta-1}\left[1-\frac{\alpha(1-\lambda) \tau}{x}\right]^{\frac{\mu}{1-\lambda}} \sum_{n=0}^{\infty} \frac{B_{p}(\gamma+n, c-\gamma)}{B(\gamma, c-\gamma)} \frac{(c)_{n}}{\Gamma(\rho n+\beta)} \frac{\left(\omega \tau^{\rho}\right)^{n}}{n !} d \tau \\
& =x^{\mu} \sum_{n=0}^{\infty} \frac{B_{p}(\gamma+n, c-\gamma)}{B(\gamma, c-\gamma)} \frac{(c)_{n}}{\Gamma(\rho n+\beta)} \frac{(\omega)^{n}}{n !} \int_{0}^{\left[\frac{x}{\alpha(1-\lambda)}\right]} \tau^{\beta+\rho n-1}\left[1-\frac{\alpha(1-\lambda) \tau}{x}\right]^{\frac{\mu}{1-\lambda}} d \tau \\
& =x^{\mu} \sum_{n=0}^{\infty} \frac{B_{p}(\gamma+n, c-\gamma)}{B(\gamma, c-\gamma)} \frac{(c)_{n}}{\Gamma(\rho n+\beta)} \frac{(\omega)^{n}}{n !}\left(\frac{x}{\alpha(1-\lambda)}\right)^{\rho n+\beta} \frac{\Gamma\left(1+\frac{\mu}{1-\lambda}\right) \Gamma(\rho n+\beta)}{\Gamma\left(\rho n+\beta+1+\frac{\mu}{1-\lambda}\right)} \\
& =\frac{x^{\mu+\beta} \Gamma\left(1+\frac{\mu}{1-\lambda}\right)}{[\alpha(1-\lambda)]^{\beta}} \sum_{n=0}^{\infty} \frac{B_{p}(\gamma+n, c-\gamma)}{B(\gamma, c-\gamma)} \frac{(c)_{n}}{\Gamma\left(\rho n+\beta+1+\frac{\mu}{1-\lambda}\right)} \frac{\left(\omega\left(\frac{x}{\alpha(1-\lambda)}\right)^{\rho}\right)^{n}}{n !} \\
& =\frac{x^{\mu+\beta} \Gamma\left(1+\frac{\mu}{1-\lambda}\right)}{[\alpha(1-\lambda)]^{\beta}} E_{\rho, \beta+1+\frac{\mu}{1-\lambda}}^{\gamma ; c}\left(\omega\left(\frac{x}{\alpha(1-\lambda)}\right)^{\rho} ; p\right),
\end{aligned}
$$

which completes the required proof.

Corollary 1 If $p=0$, then (16) leads to the following result of Mittag-Leffler function (see [36]):

$$
P_{0+}^{\mu, \lambda}\left[\tau^{\beta-1} E_{\rho, \beta}^{\gamma ; c}\left[\left(\omega \tau^{\rho}\right)\right]\right](x)=\frac{x^{\mu+\beta} \Gamma\left(1+\frac{\mu}{1-\lambda}\right)}{[\alpha(1-\lambda)]^{\beta}} E_{\rho, \beta+1+\frac{\mu}{1-\lambda}}^{\gamma}\left(\omega\left(\frac{x}{\alpha(1-\lambda)}\right)^{\rho}\right) .
$$

Corollary 2 If $\delta=1, \alpha=1, \lambda=0$ and $\mu=\mu-1$, then (16) leads to the following fractional integral formula of extended Mittag-Leffler function (see [44]):

$$
I_{a+}^{\mu}\left[\tau^{\beta-1} E_{\alpha, \beta}^{\gamma}\left(\omega(\tau)^{\rho}, p\right)\right](x)=\Gamma(\mu) x^{\mu+\beta} E_{\rho, \beta+\mu}^{\gamma}\left(\omega(x)^{\rho}, p\right) .
$$

Corollary 3 If we set $p=0$ in (18), then it leads to the following well-known result of MittagLeffler function (see [7]):

$$
I_{a+}^{\mu}\left[\tau^{\beta-1} E_{\alpha, \beta}^{\gamma}\left(\omega(\tau)^{\rho}\right)\right](x)=\Gamma(\mu) x^{\mu+\beta} E_{\rho, \beta+\mu}^{\gamma}\left(\omega(x)^{\rho}\right) .
$$

Now, we derive the following result by assuming the case that $\lambda>1$ and using equation (14).

Theorem 2 Suppose that $\rho ; \beta ; \gamma ; c ; \mu \in \mathbb{C} ;\{\Re(\rho) ; \Re(\beta) ; \Re(\mu) ; \Re(c)\}>0 ; \Re\left(\frac{\mu}{1-\lambda}\right)>-1 ; \lambda>1$; $p \geq 0$ and $\omega \in \mathbb{R}$. Then the following formula holds true:

$$
P_{0+}^{\mu, \lambda}\left[\tau^{\beta-1} E_{\rho, \beta}^{\gamma ; c}\left[\left(\omega \tau^{\rho}\right) ; p\right]\right](x)=\frac{\Gamma\left(1-\frac{\mu}{\lambda-1}\right) x^{\mu+\beta}}{[-\alpha(\lambda-1)]^{\beta}} E_{\rho, \beta+\left(1-\frac{\mu}{\lambda-1}\right)}^{\gamma ; c}\left[\omega\left(\frac{x}{-\alpha(\lambda-1)}\right)^{\rho} ; p\right] .
$$

Proof By using (6) and (12), we have

$$
\begin{aligned}
& P_{0+}^{\mu, \lambda} {\left[\tau^{\beta-1} E_{\rho, \beta}^{\gamma ; c}\left[\left(\omega \tau^{\rho}\right) ; p\right]\right](x) } \\
&\left.\quad=x^{\mu} \int_{0}^{[-\alpha(1-\lambda)}\right] \tau^{\beta-1}\left[1+\frac{\alpha(\lambda-1) \tau}{x}\right]^{\frac{\mu}{-(\lambda-1)}}\left[E_{\rho, \beta}^{\gamma ; c}\left[\left(\omega \tau^{\rho}\right) ; p\right]\right] d \tau .
\end{aligned}
$$


For convenience, we denote the right-hand side of the above equation by $I_{2}$, then

$$
I_{2}=x^{\mu} \int_{0}^{\left[\frac{x}{-\alpha(1-\lambda)}\right]} \tau^{\beta-1}\left[1+\frac{\alpha(\lambda-1) \tau}{x}\right]^{\frac{\mu}{-(\lambda-1)}} \sum_{n=0}^{\infty} \frac{B_{p}(\gamma+n, c-\gamma)}{B(\gamma, c-\gamma)} \frac{(c)_{n}}{\Gamma(\rho n+\beta)} \frac{\left(\omega \tau^{\rho}\right)^{n}}{n !} d \tau .
$$

By interchanging the order of integration and summation, we obtain

$$
I_{2}=x^{\mu} \sum_{n=0}^{\infty} \frac{B_{p}(\gamma+n, c-\gamma)}{B(\gamma, c-\gamma)} \frac{(c)_{n}}{\Gamma(\rho n+\beta)} \frac{\omega^{n}}{n !} \int_{0}^{\left[\frac{x}{-\alpha(1-\lambda)}\right]} \tau^{\beta+\rho n-1}\left[1+\frac{\alpha(\lambda-1) \tau}{x}\right]^{\frac{\mu}{-(\lambda-1)}} d \tau
$$

By substituting $\frac{-\alpha(\lambda-1) \tau}{x}=u$ and using the definitions of beta function (8) and (10) in the above equation, we get

$$
\begin{aligned}
I_{2}= & \frac{x^{\mu+\beta}}{[-\alpha(\lambda-1)]^{\beta}} \sum_{n=0}^{\infty} \frac{B_{p}(\gamma+n, c-\gamma)}{B(\gamma, c-\gamma)} \frac{(c)_{n}}{\Gamma(\rho n+\beta)} \frac{\left(\omega\left(\frac{x}{-\alpha(\lambda-1)}\right)^{\rho}\right)^{n}}{n !} \\
& \times \frac{\Gamma\left(1-\frac{\mu}{\lambda-1}\right) \Gamma(\rho n+\beta)}{\Gamma\left(\rho n+\beta+1-\frac{\mu}{\lambda-1}\right)} \\
= & \frac{x^{\mu+\beta} \Gamma\left(1-\frac{\mu}{\lambda-1}\right)}{[-\alpha(\lambda-1)]^{\beta}} \sum_{n=0}^{\infty} \frac{B_{p}(\gamma+n, c-\gamma)}{B(\gamma, c-\gamma)} \frac{(c)_{n}}{\Gamma\left(\rho n+\beta+1-\frac{\mu}{\lambda-1}\right)} \frac{\left(\omega\left(\frac{x}{-\alpha(\lambda-1)}\right)^{\rho}\right)^{n}}{n !} \\
= & \frac{x^{\mu+\beta} \Gamma\left(1-\frac{\mu}{\lambda-1}\right)}{[-\alpha(\lambda-1)]^{\beta}} E_{\rho, \beta+\left(1-\frac{\mu}{\lambda-1}\right)}^{\gamma ; c}\left[\omega\left(\frac{x}{-\alpha(\lambda-1)}\right)^{\rho} ; p\right],
\end{aligned}
$$

which completes the required proof of (20).

Corollary 4 If $p=0$, then (20) leads to the following result of Mittag-Leffler function recently introduced by Nair [36]:

$$
P_{0+}^{\mu, \lambda}\left[\tau^{\beta-1} E_{\rho, \beta}^{\gamma}\left[\left(\omega \tau^{\rho}\right)\right]\right](x)=\frac{\Gamma\left(1-\frac{\mu}{\lambda-1}\right) x^{\mu+\beta}}{[-\alpha(\lambda-1)]^{\beta}} E_{\rho, \beta+\left(1-\frac{\mu}{\lambda-1}\right)}^{\gamma}\left[\omega\left(\frac{x}{-\alpha(\lambda-1)}\right)^{\rho}\right] .
$$

Corollary 5 Again, if $\delta=1, \alpha=1, \lambda=0$ and $\mu=\mu-1$, then (21) leads to the fractional integral formula of extended Mittag-Leffler function as defined in (18).

Corollary 6 If we set $p=0$ in Corollary 5 , then it leads to the fractional integral formula of Mittag-Leffler function as defined in (19).

\section{Pathway fractional integral operator of an extended generalized Mittag-Leffler function}

In this section, we present the composition of pathway fractional integral operator associated with an extended form of the generalized Mittag-Leffler function as defined in (11).

Theorem 3 Suppose that $\rho ; \beta ; \gamma ; c ; \mu \in \mathbb{C} ;\{\Re(\rho) ; \Re(\beta) ; \Re(\mu) ; \Re(c)\}>0 ; \Re\left(\frac{\mu}{1-\lambda}\right)>-1 ; \lambda<1$; $p \geq 0 ; q>0$ and $\omega \in \mathbb{R}$. Then the following formula holds true:

$$
P_{0+}^{\mu, \lambda}\left[\tau^{\beta-1} E_{\rho, \beta}^{\gamma, q ; c}\left[\left(\omega \tau^{\rho}\right) ; p\right]\right](x)=\frac{\Gamma\left(1+\frac{\mu}{1-\lambda}\right) x^{\mu+\beta}}{[\alpha(1-\lambda)]^{\beta}} E_{\rho, \beta+\left(1+\frac{\mu}{1-\lambda}\right)}^{\gamma, q ;}\left[\omega\left(\frac{x}{\alpha(1-\lambda)}\right)^{\rho} ; p\right] .
$$


Proof By using (11) and (12), we have

$$
P_{0+}^{\mu, \lambda}\left[\tau^{\beta-1} E_{\rho, \beta}^{\gamma, q ; c}\left[\left(\omega \tau^{\rho}\right) ; p\right]\right](x)=x^{\mu} \int_{0}^{\left[\frac{x}{\alpha(1-\lambda)}\right]} \tau^{\beta-1}\left[1-\frac{\alpha(1-\lambda) \tau}{x}\right]^{\frac{\mu}{1-\lambda}} E_{\rho, \beta}^{\gamma, q ; c}\left(\omega \tau^{\rho} ; p\right) d \tau
$$

For simplicity, denote the right-hand side of the above equation by $I_{3}$, we have

$$
I_{3}=x^{\mu} \int_{0}^{\left[\frac{x}{\alpha(1-\lambda)}\right]} \tau^{\beta-1}\left[1-\frac{\alpha(1-\lambda) \tau}{x}\right]^{\frac{\mu}{1-\lambda}} \sum_{n=0}^{\infty} \frac{B_{p}(\gamma+n q, c-\gamma)}{B(\gamma, c-\gamma)} \frac{(c)_{n q}}{\Gamma(\rho n+\beta)} \frac{\left(\omega \tau^{\rho}\right)^{n}}{n !} d \tau .
$$

By interchanging the order of integration and summation, we obtain the following:

$$
\begin{aligned}
I_{3} & =x^{\mu} \sum_{n=0}^{\infty} \frac{B_{p}(\gamma+n q, c-\gamma)}{B(\gamma, c-\gamma)} \frac{(c)_{n q}}{\Gamma(\rho n+\beta)} \frac{(\omega)^{n}}{n !} \int_{0}^{\left[\frac{x}{\alpha(1-\lambda)}\right]} \tau^{\beta+\rho n-1}\left[1-\frac{\alpha(1-\lambda) \tau}{x}\right]^{\frac{\mu}{1-\lambda}} d \tau \\
& =x^{\mu} \sum_{n=0}^{\infty} \frac{B_{p}(\gamma+n q, c-\gamma)}{B(\gamma, c-\gamma)} \frac{(c)_{n q}}{\Gamma(\rho n+\beta)} \frac{(\omega)^{n}}{n !}\left(\frac{x}{\alpha(1-\lambda)}\right)^{\rho n+\beta} \frac{\Gamma\left(1+\frac{\mu}{1-\lambda}\right) \Gamma(\rho n+\beta)}{\Gamma\left(\rho n+\beta+1+\frac{\mu}{1-\lambda}\right)} \\
& =\frac{x^{\mu+\beta} \Gamma\left(1+\frac{\mu}{1-\lambda}\right)}{[\alpha(1-\lambda)]^{\beta}} \sum_{n=0}^{\infty} \frac{B_{p}(\gamma+n q, c-\gamma)}{B(\gamma, c-\gamma)} \frac{(c)_{n q}}{\Gamma\left(\rho n+\beta+1+\frac{\mu}{1-\lambda}\right)} \frac{\left(\omega\left(\frac{x}{\alpha(1-\lambda)}\right)^{\rho}\right)^{n}}{n !} \\
& =\frac{x^{\mu+\beta} \Gamma\left(1+\frac{\mu}{1-\lambda}\right)}{[\alpha(1-\lambda)]^{\beta}} E_{\rho, \beta+1+\frac{\mu}{1-\lambda}}^{\gamma, q ; c}\left(\omega\left(\frac{x}{\alpha(1-\lambda)}\right)^{\rho} ; p\right),
\end{aligned}
$$

which completes the required proof.

Corollary 7 If $p=0$, and $q=1$, then (22) leads to the following result of Mittag-Leffler function (see [36]):

$$
\begin{aligned}
P_{0+}^{\mu, \lambda}\left[\tau^{\beta-1} E_{\rho, \beta}^{\gamma}\left[\left(\omega \tau^{\rho}\right)\right]\right](x) \\
\quad=\frac{x^{\mu+\beta} \Gamma\left(1+\frac{\mu}{1-\lambda}\right)}{[\alpha(1-\lambda)]^{\beta}} E_{\rho, \beta+1+\frac{\mu}{1-\lambda}}^{\gamma}\left(\omega\left(\frac{x}{\alpha(1-\lambda)}\right)^{\rho}\right) .
\end{aligned}
$$

Corollary 8 If $\alpha=1, \lambda=0$ and $\mu=\mu-1$, then (22) leads to the following result of extended Mittag-Leffler function (see [45]):

$$
P_{0+}^{\mu-1,0}\left[\tau^{\beta-1} E_{\rho, \beta}^{\gamma, q}\left[\left(\omega \tau^{\rho}\right) ; p\right]\right](x)=\Gamma(\mu) x^{\mu+\beta} E_{\rho, \beta+\mu}^{\gamma, q}\left(\omega(x)^{\rho} ; p\right) .
$$

Remark 1 If we set $p=0$ in Corollary 8, then we get the fractional integrals of MittagLeffler function earlier proved in [21].

Now, we derive the following result by assuming the case that $\lambda>1$.

Theorem 4 Suppose that $\rho ; \beta ; \gamma ; c ; \mu \in \mathbb{C} ;\{\Re(\rho) ; \Re(\beta) ; \Re(\mu) ; \Re(c)\}>0 ; \Re\left(\frac{\mu}{1-\lambda}\right)>-1 ; \lambda>1$; $p \geq 0 ; q>0$ and $\omega \in \mathbb{R}$. Then the following formula holds true:

$$
P_{0+}^{\mu, \lambda}\left[\tau^{\beta-1} E_{\rho, \beta}^{\gamma, q ; c}\left[\left(\omega \tau^{\rho}\right) ; p\right]\right](x)=\frac{\Gamma\left(1-\frac{\mu}{\lambda-1}\right) x^{\mu+\beta}}{[-\alpha(\lambda-1)]^{\beta}} E_{\rho, \beta+\left(1-\frac{\mu}{\lambda-1}\right)}^{\gamma, q ; c}\left[\omega\left(\frac{x}{-\alpha(\lambda-1)}\right)^{\rho} ; p\right] .
$$


Proof By using (11) and (14), we obtain

$$
\begin{aligned}
& P_{0+}^{\mu, \lambda}\left[\tau^{\beta-1} E_{\rho, \beta}^{\gamma, q ; c}\left[\left(\omega \tau^{\rho}\right) ; p\right]\right](x) \\
& \quad=x^{\mu} \int_{0}^{\left[\frac{x}{-\alpha(\lambda-1)}\right]} \tau^{\beta-1}\left[1+\frac{\alpha(\lambda-1) \tau}{x}\right]^{\frac{\mu}{-(\lambda-1)}}\left[E_{\rho, \beta}^{\gamma, q ; c}\left[\left(\omega \tau^{\rho}\right) ; p\right]\right] d \tau .
\end{aligned}
$$

For convenience, we denote the right-hand side of the above equation by $I_{2}$, then

$$
I_{4}=x^{\mu} \int_{0}^{\left[\frac{x}{-\alpha(\lambda-1)}\right]} \tau^{\beta-1}\left[1+\frac{\alpha(\lambda-1) \tau}{x}\right]^{\frac{\mu}{-(\lambda-1)}} \sum_{n=0}^{\infty} \frac{B_{p}(\gamma+n q, c-\gamma)}{B(\gamma, c-\gamma)} \frac{(c)_{n q}}{\Gamma(\rho n+\beta)} \frac{\left(\omega \tau^{\rho}\right)^{n}}{n !} d \tau .
$$

By interchanging the order of integration and summation, we obtain

$$
I_{4}=x^{\mu} \sum_{n=0}^{\infty} \frac{B_{p}(\gamma+n q, c-\gamma)}{B(\gamma, c-\gamma)} \frac{(c)_{n q}}{\Gamma(\rho n+\beta)} \frac{\omega^{n}}{n !} \int_{0}^{\left[\frac{x}{-\alpha(\lambda-1)}\right]} \tau^{\beta+\rho n-1}\left[1+\frac{\alpha(\lambda-1) \tau}{x}\right]^{\frac{\mu}{-(\lambda-1)}} d \tau .
$$

By substituting $\frac{-\alpha(\lambda-1) \tau}{x}=u$ and using the definitions of beta function (8) and (10) in the above equation, we obtain

$$
\begin{aligned}
I_{4}= & \frac{x^{\mu+\beta}}{[-\alpha(\lambda-1)]^{\beta}} \sum_{n=0}^{\infty} \frac{B_{p}(\gamma+n q, c-\gamma)}{B(\gamma, c-\gamma)} \frac{(c)_{n q}}{\Gamma(\rho n+\beta)} \frac{\left(\omega\left(\frac{x}{-\alpha(\lambda-1)}\right)^{\rho}\right)^{n}}{n !} \\
& \times \frac{\Gamma\left(1-\frac{\mu}{\lambda-1}\right) \Gamma(\rho n+\beta)}{\Gamma\left(\rho n+\beta+1-\frac{\mu}{\lambda-1}\right)} \\
= & \frac{x^{\mu+\beta} \Gamma\left(1-\frac{\mu}{\lambda-1}\right)}{[-\alpha(\lambda-1)]^{\beta}} \sum_{n=0}^{\infty} \frac{B_{p}(\gamma+n q, c-\gamma)}{B(\gamma, c-\gamma)} \frac{(c)_{n q}}{\Gamma\left(\rho n+\beta+1-\frac{\mu}{\lambda-1}\right)} \frac{\left(\omega\left(\frac{x}{-\alpha(\lambda-1)}\right)^{\rho}\right)^{n}}{n !} \\
= & \frac{x^{\mu+\beta} \Gamma\left(1-\frac{\mu}{\lambda-1}\right)}{[-\alpha(\lambda-1)]^{\beta}} E_{\rho, \beta+\left(1-\frac{\mu}{\lambda-1}\right)}^{\gamma, q ; c}\left[\omega\left(\frac{x}{-\alpha(\lambda-1)}\right)^{\rho} ; p\right]
\end{aligned}
$$

which completes the required proof of (25).

Corollary 9 If $p=0$ and $q=1$, then (25) leads to the following result of Mittag-Leffler function recently introduced by Nair [36]:

$$
P_{0+}^{\mu, \lambda}\left[\tau^{\beta-1} E_{\rho, \beta}^{\gamma}\left[\left(\omega \tau^{\rho}\right) ; p\right]\right](x)=\frac{\Gamma\left(1-\frac{\mu}{\lambda-1}\right) x^{\mu+\beta}}{[-\alpha(\lambda-1)]^{\beta}} E_{\rho, \beta+\left(1-\frac{\mu}{\lambda-1}\right)}^{\gamma}\left[\omega\left(\frac{x}{-\alpha(\lambda-1)}\right)^{\rho}\right]
$$

Corollary 10 If $\alpha=1, \lambda=0$ and $\mu=\mu-1$, then (25) leads to the result of fractional integral containing an extended Mittag-Leffler function in its kernel defined in Corollary 8.

\section{Conclusion}

In this paper, we have presented two pathway fractional integration formulae associated with extended Mittag-Leffler functions. The obtained result provided extended forms of the known results earlier proved by Nair [36]. 
Authors' contributions

The authors have contributed equally to this manuscript. They read and approved the final manuscript.

\section{Author details}

${ }^{1}$ Department of Mathematics, International Islamic University, Islamabad, Pakistan. ${ }^{2}$ Department of Mathematical Sciences, BUITEMS, Quetta, Pakistan. ${ }^{3}$ Department of Mathematics, University of Sargodha, Sargodha, Pakistan. ${ }^{4}$ Department of Mathematics, Gomal University, Dera Ismail Khan, Pakistan.

\section{Publisher's Note}

Springer Nature remains neutral with regard to jurisdictional claims in published maps and institutional affiliations.

Received: 10 December 2016 Accepted: 9 June 2017 Published online: 20 June 2017

\section{References}

1. Băleanu, D, Diethelm, K, Scalas, E, Trujillo, JJ: Fractional Calculus: Models and Numerical Methods, pp. 10-16. World Scientific, Singapore (2012)

2. Nisar, KS, Purohit, SD, Mondal, SR: Generalized fractional kinetic equations involving generalized Struve function of the first kind. J. King Saud Univ., Sci. 28, 167-171 (2016)

3. Purohit, SD: Solutions of fractional partial differential equations of quantum mechanics. Adv. Appl. Math. Mech. 5 639-651 (2013)

4. Podlubny, I: Fractional Differential Equations: An Introduction to Fractional Derivatives, Fractional Differential Equations, to Methods of Their Solution and Some of Their Applications. Academic Press, San Diego (1999)

5. Uchaikin, W: Fractional Derivatives for Physicists and Engineers. Volume I: Background and Theory, Volume II: Applications. Nonlinear Physical Science. Springer, Berlin (2013)

6. Džrbašjan, MM: Integral Transforms and Representations of Functions in the Complex Domain. Nauka, Moscow (1966) (in Russian)

7. Kilbas, AA, Saigo, M: On Mittag-Leffler type function, fractional calculus operators and solutions of integral equations. Integral Transforms Spec. Funct. 4, 355-370 (1996)

8. Gorenflo, R, Mainardi, F: Fractional calculus: integral and differential equations of fractional order. In: Carpinteri, A, Mainardi, F (eds.) Fractals and Fractional Calculus in Continuum Mechanics. Springer Series on CSM Courses and Lectures, vol. 378, pp. 223-276. Springer, Wien (1997)

9. Gorenflo, R, Mainardi, F, Srivastava, HM: Special functions in fractional relaxation-oscillation and fractional diffusion-wave phenomena. In: Bainov, D (ed.) Proceedings of the Eighth International Colloquium on Differentia Equations, pp. 195-202. VSP Publishers, Utrecht (1998)

10. Gorenflo, R, Luchko, Y, Mainardi, F: Wright functions as scale-invariant solutions of the diffusion-wave equation J. Comput. Appl. Math. 118, 175-191 (2000)

11. Kilbas, AA, Srivastava, HM, Trujillo, JJ: Theory and Applications of Fractional Differential Equations. North-Holland Mathematical Studies, vol. 204. Elsevier, Amsterdam (2006)

12. Saigo, M, Kilbas, AA: On Mittag-Leffler type function and applications. Integral Transforms Spec. Funct. 7, 97-112 (1998)

13. Hilfer, R, Seybold, $\mathrm{H}$ : Computation of the generalized Mittag-Leffler function and its inverse in the complex plane. Integral Transforms Spec. Funct. 17, 637-652 (2006)

14. Seybold, HJ, Hilfer, R: Numerical results for the generalized Mittag-Leffler function. Fract. Calc. Appl. Anal. 8, 127-139 (2005)

15. Prabhakar, TR: A singular integral equation with a generalized Mittag-Leffler function in the kernel. Yokohama Math. J. 19, 7-15 (1971)

16. Gorenflo, R, Kilbas, AA, Rogosin, SV: On the generalized Mittag-Leffler type functions. Integral Transforms Spec. Funct. 7, 215-224 (1998)

17. Srivastava, HM: A contour integral involving Fox's H-function. Indian J. Math. 14, 1-6 (1972)

18. Srivastava, HM: A note on the integral representation for the product of two generalized Rice polynomials. Collect. Math. 24, 117-121 (1973)

19. Srivastava, HM, Gupta, KC, Goyal, SP: The H-Functions of One and Two Variables with Applications. South Asian Publishers, New Delhi (1982)

20. Srivastava, HM, Joshi, CM: Integral representation for the product of a class of generalized hypergeometric polynomials. Acad. Roy. Belg. Bull. Cl. Sci. (Ser. 5) 60, 919-926 (1974)

21. Srivastava, HM, Tomovski, Ž: Fractional calculus with an integral operator containing a generalized Mittag-Leffler function in the kernel. Appl. Math. Comput. 211, 189-210 (2009)

22. Özarslan, MA, YıImaz, B: The extended Mittag-Leffler function and its properties. J. Inequal. Appl. 2014, Article ID 85 (2014)

23. Chaudhry, MA, Qadir, A, Srivastava, HM, Paris, RB: Extended hypergeometric and confluent hypergeometric functions. Appl. Math. Comput. 159, 589-602 (2004)

24. Mainardi, F: On some properties of the Mittag-Leffler function, $E_{\alpha}(t z)$, completely monotone for $t>0$ with $0<\alpha<1$. arXiv:1305.0161

25. Mittal, E, Pandey, RM, Joshi, S: On extension of Mittag-Leffler function. Appl. Appl. Math. 11(1), 307-316 (2016)

26. Băleanu, D, Mustafa, OG: On the global existence of solutions to a class of fractional differential equations. Comput. Math. Appl. 59, 1835-1841 (2010)

27. Chouhan, A, Purohit, SD, Saraswat, S: An alternative method for solving generalized differential equations of fractional order. Kragujev. J. Math. 37(2), 299-306 (2013)

28. Kumar, D, Purohit, SD, Choi, J: Generalized fractional integrals involving product of multivariable $\mathrm{H}$-function and a general class of polynomials. J. Nonlinear Sci. Appl. 9(1), 8-21 (2016) 
29. Purohit, SD, Kalla, SL: On fractional partial differential equations related to quantum mechanics. J. Phys. A, Math. Theor. 44(4), Article ID 045202 (2011)

30. Băleanu, D, Kumar, D, Purohit, SD: Generalized fractional integrals of product of two H-functions and a general class of polynomials. Int. J. Comput. Math. 93(8), 1320-1329 (2016)

31. Purohit, SD, Kalla, SL, Suthar, DL: Fractional integral operators and the multiindex Mittag-Leffler function. Scientia, Ser. A, Math. Sci. 21, 87-96 (2011)

32. Yang, XJ, Tenreiro Machado, JA Baleanu, D, Cattani, C: On exact traveling-wave solutions for local fractional Korteweg-de Vries equation. Chaos, Interdiscip. J. Nonlinear Sci. 26(8), Article ID 084312 (2016)

33. Yang, XJ: Fractional derivatives of constant and variable orders applied to anomalous relaxation models in heat-transfer problems. Therm. Sci. (2016). doi:10.2298/TSCI161216326Y

34. Yang, XJ, Gao, F, Tenreiro Machado, JA, Baleanu, D: A new fractional derivative without singular kernel: application to the modelling of the steady heat flow. Therm. Sci. 20, $753-756$ (2016)

35. Yang, XJ, Tenreiro Machado, JA, Cattani, C, Gao, F: On a fractal LC-electric circuit modeled by local fractional calculus. Commun. Nonlinear Sci. Numer. Simul. 47, 200-206 (2017)

36. Nair, SS: Pathway fractional integration operator. Fract. Calc. Appl. Anal. 12, 237-252 (2009)

37. Mathai, AM: A pathway to matrix-variate gamma and normal densities. Linear Algebra Appl. 396, 317-328 (2005)

38. Mathai, AM, Haubold, HJ: Pathway model, superstatistics, Tsallis statistics and a generalize measure of entropy. Physica A 375, 110-122 (2007)

39. Mathai, AM, Haubold, HJ: On generalized distributions and path-ways. Phys. Lett. A 372, 2109-2113 (2008)

40. Băleanu, D, Agarwal, P: A composition formula of the pathway integral transform operator. Note Mat. 34, 145-155 (2014)

41. Nisar, KS, Mondal, SR, Agarwal, P: Pathway fractional integral operator associated with Struve function of first kind. Adv. Stud. Contemp. Math. 26, 63-70 (2016)

42. Agarwal, P, Purohit, SD: The unified pathway fractional integral formulae. J. Fract. Calc. Appl. 4(1), 105-112 (2013)

43. Nisar, KS, Purohit, SD, Abouzaid, MS, Al-Qurashi, M, Baleanu, D: Generalized k-Mittag-Leffler function and its composition with pathway integral operators. J. Nonlinear Sci. Appl. 9, 3519-3526 (2016)

44. Rahman, G, Agarwal, P, Mubeen, S, Arshad, M: Fractional integral operators involving extended Mittag-Leffler function as its kernel. Bol. Soc. Mat. Mexicana (2017). doi:10.1007/s40590-017-0167-5

45. Rahman, G, Băleanu, D, Al-Qurashi, M, Purohit, SD, Mubeen, S, Arshad, M: Extended Mittag-Leffler function via fractional calculus. J. Nonlinear Sci. Appl. (2017, accepted)

\section{Submit your manuscript to a SpringerOpen ${ }^{\circ}$ journal and benefit from:}

- Convenient online submission

- Rigorous peer review

- Open access: articles freely available online

- High visibility within the field

- Retaining the copyright to your article

Submit your next manuscript at $\boldsymbol{\Delta}$ springeropen.com 